\title{
Cultura de segurança do paciente em um hospital acreditado de alta complexidade
}

\author{
Culture of patient safety in an accredited high complexity hospital \\ Cultura de seguridad del paciente en un hospital acreditado de alta complejidad
}

Recebido: 15/05/2021 | Revisado: 23/05/2021 | Aceito: 26/05/2021 | Publicado: 11/06/2021

\author{
Magda Macedo Madalozzo \\ ORCID: https://orcid.org/0000-0002-1633-8175 \\ Universidade de Caxias do Sul, Brasil \\ E-mail:mmmadalo@ucs.br \\ João Ignacio Pires Lucas \\ ORCID: https://orcid.org/0000-0002-6307-1338 \\ Universidade de Caxias do Sul, Brasil \\ E-mail: jiplucas@ucs.br \\ Lilia Aparecida Kanan \\ ORCID: https://orcid.org/0000-0001-6412-0544 \\ Universidade do Planalto Catarinense, Brasil \\ E-mail: lilia.kanan@gmail.com \\ Silvana Regina Ampessan Marcon \\ ORCID: https://orcid.org/0000-0002-7726-9900 \\ Universidade de Caxias do Sul, Brasil \\ E-mail: sramarco@ucs.br \\ Aline Santos de Souza \\ ORCID: https://orcid.org/0000-0002-7173-6870 \\ Universidade de Caxias do Sul, Brasil \\ E-mail: assouza@ucs.br \\ Fernanda Tonietto Michelin \\ ORCID: https://orcid.org/0000-0002-2546-4384 \\ Universidade de Caxias do Sul, Brasil \\ E-mail: ftmichelin@ucs.br \\ Scheila Pacheo Hoffman \\ ORCID: https://orcid.org/0000-0002-9339-3629 \\ Universidade de Caxias do Sul, Brasil \\ E-mail: scheila.hoffman@ucs.br
}

\begin{abstract}
Resumo
O propósito do artigo é apresentar os resultados de pesquisa sobre segurança do paciente a partir das percepções dos funcionários de uma instituição hospitalar de alta complexidade, localizada na região sul do Brasil. Trata-se de pesquisa aplicada, transversal, exploratória e descritiva, com design survey e abordagem quantitativa. À coleta de dados foi utilizado o Hospital Survey on Patient Safety Culture (HSOPSC), desenvolvido pela Agency for Healthcare Research And Quality e validado no Brasil por Reis (2013). Participaram da pesquisa 646 (67\%) entre os 960 funcionários do hospital. Como principais resultados do estudo foram identificadas como dimensões fortes para a segurança dos pacientes 'aprendizado organizacional', 'apoio da gestão hospitalar para segurança do paciente', 'frequência de eventos relatados' e 'expectativas sobre o seu supervisor/chefe' e 'ações promotoras de segurança do paciente. De outro modo, as dimensões 'passagem de plantão/turno' e 'respostas não punitivas aos erros' caracterizaram-se como frágeis para a cultura de segurança no hospital. Portanto, reforçar os aspectos promotores e intervir nos aspectos fragilizantes representam ações urgentes e necessárias a serem adotadas no hospital em tela, assim como em todo o sistema hospitalar.
\end{abstract}

Palavras-chave: Segurança do paciente; Gestão da segurança; Cultura organizacional.

\begin{abstract}
The purpose of the article is to present the results of the research on patient safety from the perceptions of the employees of high complex hospital, located in the southern region of Brazil. It is applied, transversal, exploratory and descriptive research, with survey design and quantitative approach. Data collection was performed using the Hospital Survey on Patient Safety Culture (HSOPSC), developed by the Agency for Healthcare Research And Quality and validated in Brazil by Reis (2013). 646 (67\%) of the 960 hospital employees participated in the survey. The main results of the study were identified as strong dimensions for patient safety 'organizational learning', 'hospital management support for patient safety', 'frequency of reported events' and' expectations about your supervisor / boss' and 'actions promoting patient safety. Otherwise, the dimensions 'shift change / shift' and 'non-punitive responses to errors' were characterized as fragile for the safety culture in the hospital. Therefore, reinforcing the promoter aspects
\end{abstract}


and intervening in the weakening aspects represent urgent and necessary actions to be taken in the hospital in question, as well as in the entire hospital system.

Keywords: Patient safety; Security management; Organizational culture.

\begin{abstract}
Resumen
El propósito del artículo es presentar los resultados de la investigación sobre seguridad del paciente a partir de las percepciones de los empleados de un hospital de alta complejidad, ubicado en la región sur de Brasil. Es una investigación aplicada, transversal, exploratoria y descriptiva, con diseño de encuestas y enfoque cuantitativo. La recolección de datos se realizó mediante la Encuesta Hospitalaria sobre Cultura de Seguridad del Paciente (HSOPSC), desarrollada por la Agencia para la Investigación y la Calidad en Salud y validada en Brasil por Reis (2013). 646 (67\%) de los 960 empleados del hospital participaron en la encuesta. Los principales resultados del estudio se identificaron como dimensiones sólidas para el 'aprendizaje organizacional' de la seguridad del paciente, el 'apoyo de la gestión hospitalaria para la seguridad del paciente', la 'frecuencia de los eventos informados' y las 'expectativas sobre su supervisor / jefe' y 'acciones que promuevan la seguridad del paciente'. De lo contrario, las dimensiones "turno turno / turno" y "respuestas no punitivas a los errores" se caracterizaron como frágiles para la cultura de seguridad en el hospital. Por tanto, reforzar los aspectos promotores e intervenir en los aspectos debilitantes representan acciones urgentes y necesarias a adoptar en el hospital en cuestión, así como en todo el sistema hospitalario.
\end{abstract}

Palabras clave: Seguridad del paciente; Gestion de seguridad; Cultura de la organización.

\title{
1. Introdução
}

O interesse na segurança do paciente está crescendo exponencialmente, alimentado por pesquisas epidemiológicas que revelam a extensão da assistência insegura (Simsekler, Qaz, Alalamia, Ellahham \& Ozonoff; 2020). A Organização Mundial de Saúde - OMS - registra que danos à saúde acometem dezenas de milhares de pessoas anualmente, em todos os países (Paes, Braz, Sauthier, Oliveira, \& Stipp, 2020).

Apesar de números preocupantes em relação a eventos adversos e segurança do paciente, evidencia-se a qualidade em saúde como algo elementar, porém essencialmente crítico (Oliveira et al., 2020). Por conta disto a Organização Mundial de Saúde (WHO) incluiu a segurança como um dos preceitos básicos para o atendimento qualificado, além da eficácia, eficiência, acessibilidade, aceitabilidade, equidade e cuidados com a segurança dos funcionários (WHO, 2006).

A manutenção e melhoria constante dos cuidados em saúde, se estende além da simples implementação de ações isoladas de técnicas multidisciplinares. Implicam instâncias institucionais e organizacionais. A sustentação de práticas para promover a segurança dos pacientes transita pelo fortalecimento de valores e crenças de cuidado efetivo que precisam ser incorporados como cultura de segurança do paciente. Isso significa que o cuidado com o paciente deverá ser expresso de modo natural nos comportamentos e ações cotidianas dos diferentes profissionais que trabalham em instituições de saúde, fundamentados no uso otimizado de recursos disponíveis, na maior satisfação dos clientes internos e externos e na redução de riscos associados ao cuidado (Madalozzo \& Zanelli, 2016; Oliveira et al, 2020; Reis, 2013). Assim, transformações culturais precisam ser acompanhadas por mudanças estruturais e procedimentais (Simsekler et al., 2020).

No Brasil, os processos de acreditação hospitalar sugeridos pela Organização Nacional de Acreditação (ONA), organização não governamental, sem fins lucrativos, criada para certificar a qualidade de serviços de saúde, têm se mostrado eficientes para fortalecer a cultura de segurança do paciente como resultado da implementação de protocolos e estratégias de segurança assistencial. Por conta disso amplia-se o número de instituições hospitalares brasileiras acreditadas: entre 2015 e 2019 este número é de 3270 instituições e entre 2018 e 2019 houve um incremento de 21\% nas acreditações (ONA, 2020). Em consequência ampliou-se a necessidade de identificar indicadores para conhecer aspectos que evidenciam a cultura de segurança do paciente e todas as melhorias realizadas nos processos de trabalho ao longo dos diferentes níveis de acreditação.

Um dos instrumentos mais utilizados em diferentes países para mensurar a cultura de segurança do paciente, é o Hospital Survey on Patient Safety Culture (HSOPSC), que foi desenvolvido pela Agency for Healthcare Research And Quality, (AHRQ) e validado no Brasil por Reis (2013). Esse artigo descreve os resultados de uma pesquisa fundamentada na pergunta 
'quais as percepções dos funcionários sobre cultura de segurança do paciente em uma instituição hospitalar acreditado de alta complexidade'?

A cultura de segurança do paciente pode ser compreendida como um processo que enfatiza políticas, sistemas e procedimentos sistêmicos para garantir a segurança do paciente. Esse processo inclui aspectos técnicos, gestão e liderança eficientes de gestão de pessoas e trabalho em equipe utilizando comunicação assertiva (Im \& Park, 2018; Reis, Paiva \& Sousa, 2018). Para os autores, a cultura de segurança do paciente deve ser compartilhada e praticada por toda a equipe, incluindo enfermeiras, médicos, agentes operacionais e de apoio, gerentes hospitalares e não apenas por gerentes de segurança. Este compartilhamento precisa ocorrer para que a cultura de segurança do paciente funcione como parte de uma cultura organizacional sólida.

Andrade, Pereira e Nunes (2015) a conceituam como "um princípio e um qualificador do cuidado, concretizado a partir de medidas estruturais, organizacionais, gerenciais, de responsabilidade e formação profissional que antecipam as possibilidades de erros e, simultaneamente, garantam a satisfação do binômio paciente-profissional” (p.528). A segurança do paciente significa que o paciente não foi ferido durante o tratamento (AHRQ, 2004). Para Im e Park (2018), os acidentes de segurança do paciente são causados pela exposição a riscos, como comunicação deficiente entre a equipe de profissionais, gravidade e vulnerabilidade do paciente e danos não intencionais no processo de prestação de serviços médicos em um ambiente médico cada vez mais complexo com o rápido desenvolvimento da ciência e da tecnologia. Segurança do paciente é um conceito multidimensional que está associado a erros médicos e a eventos de (in)segurança do paciente em sistemas de prestação de cuidados de saúde (Simsekler et. al, 2020).

A segurança do paciente representa um valor fundamental e um desafio. Doo e Kim (2020) afirmam que é necessário a implementação de estratégias que permitam aos profissionais que atuam diretamente com o paciente participarem da tomada de decisão em hospitais. Em suas pesquisas estes autores identificaram que o silêncio organizacional tem um efeito direto e indireto na segurança do paciente e a satisfação com a comunicação organizacional é uma dimensão importante para a segurança do paciente.

Para promover as atividades de gestão da segurança do paciente nos hospitais, é importante desenvolver programas específicos de educação para fortalecer a cultura de segurança do paciente e o compromisso organizacional, e implementá-lo em conexão com os profissionais que possuem responsabilidades com o cuidado do paciente (Im \& Park, 2018). Conforme os autores, o conteúdo educacional pode ser composto por sistema de relatórios, gestão do ambiente de segurança, liderança, aprimoramento da cultura hospitalar e comunicação emocional, e diversos cases são apresentados para auxiliar no entendimento do assunto.

\section{Metodologia}

Trata-se de pesquisa aplicada, transversal, exploratória e descritiva, de abordagem quantitativa com design de survey. A coleta dos dados foi realizada nos meses de outubro e novembro de 2019 pela equipe de profissionais do Escritório da Qualidade de um hospital geral de alta complexidade do sul do Brasil, por meio do Google Drive. Todos os 960 funcionários foram convidados a participar.

O instrumento HSOPSC foi validado no Brasil por Reis (2013). É integrado por 54 perguntas organizadas em 12 dimensões para a análise da cultura de segurança em três grandes áreas: hospital, unidade e segurança, mais 2 itens de respostas únicas voltados à avaliação geral da segurança do paciente na unidade de trabalho do participante e à frequência de notificação de eventos adversos e/ou não conformidades que o participante da pesquisa teria preenchido nos últimos 12 meses, e, por fim, 10 perguntas para a caracterização do perfil sociodemográfico e das áreas de atuação dos participantes. Os 42 itens formulados para as 12 dimensões são formatados na escala Likert (de cinco pontos entre os graus de concordância e 
discordância) e do tipo Likert (cinco pontos de frequências crescentes do nunca ao sempre). São sete as dimensões relacionadas à Unidade: "trabalho em equipe dentro das unidades" (D1), "expectativas do supervisor/chefe e ações promotoras da segurança" (D2), "aprendizado organizacional- melhoria contínua" (D3), "adequação de profissionais" (D4), "respostas não punitivas aos erros" (D5), "retorno da informação e comunicação sobre erro" (D6) e "abertura da comunicação" (D7). As dimensões relativas ao Hospital são três: "apoio da gestão para a segurança do paciente" (D8), "trabalho em equipe entre as unidades" (D9) e "passagem de plantão/turno e transferências" (D10). Quanto a segurança são duas: "frequência de notificação de eventos" (D11) e "percepção geral da segurança do paciente" (D12). As duas perguntas com itens únicos voltadas à avaliação geral da segurança do paciente na unidade são formatadas, respectivamente, para a avaliação, com as alternativas de respostas entre o excelente, muito boa, regular, ruim e muito ruim, e para o relato da frequência de notificações adversas: com alternativas de faixas de quantidades de notificações entre o zero, 1 a 2, 3 a 5, 6 a 10, 11 a 20 e, por fim, 21 ou mais notificações. O tempo médio de aplicação foi de 15 minutos.

A análise do instrumento foi realizada com base nas técnicas da estatística descritiva e seus resultados comparados com a revisão da literatura. Para tanto foram utilizados os recursos do software IBM SPSS Statistics versão 25. O estudo foi aprovado pelo Conselho Científico e Editorial do hospital (DEPE - COEDI) e pelo Comitê de Ética em Pesquisa conforme parecer $\mathrm{n}^{\circ}$ 4.484.096, de 23 de dezembro de 2020.

\section{Resultados e Discussão}

Participaram da pesquisa 646 (67\%) do total de 960 funcionários do hospital, efetivo do período. Na Tabela 1, a seguir, é possível visualizar a caracterização da amostra.

Tabela 1. Caracterização da Amostra $(n=646)$.

\begin{tabular}{|c|c|c|c|}
\hline Variável & & $\mathrm{N}$ & $\%$ \\
\hline \multirow[t]{2}{*}{ Sexo } & Masculino & 79 & 12 \\
\hline & Feminino & 567 & 88 \\
\hline \multirow[t]{4}{*}{ Faixa Etária } & $18-35$ & 234 & 36 \\
\hline & $36-45$ & 153 & 24 \\
\hline & 47 anos ou + & 45 & 7 \\
\hline & Sem resposta & 214 & 33 \\
\hline \multirow[t]{4}{*}{ Escolaridade } & Fundamental e médio completo & 241 & 37 \\
\hline & Superior incompleto & 205 & 32 \\
\hline & Superior completo & 58 & 9 \\
\hline & Pós-Graduação & 142 & 22 \\
\hline \multirow{4}{*}{$\begin{array}{l}\text { Tempo de Trabalho no } \\
\text { Hospital }\end{array}$} & Menos de 1 anos & 168 & 26 \\
\hline & 1 a 5 anos & 278 & 43 \\
\hline & 6 a 10 anos & 122 & 19 \\
\hline & 11 anos ou + & 78 & 12 \\
\hline \multirow{4}{*}{$\begin{array}{l}\text { Tempo de trabalho na } \\
\text { Unidade }\end{array}$} & Menos de 1 anos & 203 & 31 \\
\hline & 1 a 5 anos & 288 & 45 \\
\hline & 6 a 10 anos & 100 & 16 \\
\hline & 11 anos ou + & 55 & 8 \\
\hline \multirow{2}{*}{$\begin{array}{l}\text { Contato direto com o } \\
\text { paciente }\end{array}$} & Sim & 485 & 75 \\
\hline & Não & 161 & 25 \\
\hline \multirow{3}{*}{$\begin{array}{l}\text { Carga horária de trabalho } \\
\text { por semana }\end{array}$} & $<20 \mathrm{~h}$ & 15 & 2 \\
\hline & de $20 \mathrm{~h}$ a $39 \mathrm{~h}$ & 150 & 24 \\
\hline & + de $40 \mathrm{~h}$ & 481 & 74 \\
\hline
\end{tabular}

Fonte: Dados primários. 
Observa-se na Tabela 1 que, de modo geral, o perfil dos participantes está representado por mulheres $(87,8 \%)$, na faixa de 18 a 35 anos (36\%), com ensino fundamental/médio (37\%). Trabalham no hospital entre 1 a 5 anos (43\%), têm contato direto com o paciente (75\%) e totalizam de 40 a 59 horas semanais de trabalho $(72,3 \%)$.

Quanto a área de trabalho, a maioria dos participantes $(59,3 \%)$ atua diretamente na clínica com pacientes, pois ocupam funções de médicos, enfermeiros, técnicos de enfermagem, maqueiros, seguidos de profissionais e auxiliares dos serviços de apoio como nutrição, higienização, lavanderia, cozinha e costura, farmácia, laboratório, radiologia, etc. Cerca de $26 \%$ atuam no apoio gerencial, $10 \%$ no setor administrativo, $3,3 \%$ em atividades de gestão e os demais $(0,9 \%)$ em outros setores.

A Tabela 2 apresenta a definição e as dimensões do HSOPSC e variáveis de resultados mensuráveis. A análise foi realizada conforme a estrutura do instrumento, na perspectiva das dimensões consideradas fortes, moderadas ou frágeis para a cultura de segurança do paciente, nas percepções dos respondentes do hospital. Os resultados são apresentados por blocos, de acordo com a respectiva distribuição das dimensões envolvidas em cada uma das três grandes áreas pesquisadas: nível da unidade, nível da organização hospitalar e medidas de resultados únicos e múltiplos.

De acordo com os critérios de apuração do HSOPSC, $75 \%$ é o percentual mínimo para classificar alguma dimensão pesquisada como forte da segurança do paciente. Percentuais menores de 50\% nas dimensões devem ser considerados como frágeis da segurança do paciente.

Tabela 2. Definição e Dimensões do HSOPSC e Variáveis de Resultados Mensuráveis

\begin{tabular}{|c|c|c|c|}
\hline \multicolumn{2}{|c|}{ Dimensão } & $\%$ positividade & Avaliação \\
\hline \multicolumn{4}{|c|}{ Nível da Unidade } \\
\hline D1 & $\begin{array}{l}\text { Trabalho em equipe dentro das unidades: define se os } \\
\text { profissionais apoiam uns aos outros, tratam uns aos } \\
\text { outros com respeito e trabalham juntos como uma equipe }\end{array}$ & $71 \%$ & Moderado \\
\hline D2 & $\begin{array}{l}\text { Expectativa sobre o supervisor/chefe e ações promotoras da segurança } \\
\text { do paciente: avalia se os supervisores e gerentes consideram as } \\
\text { sugestões dos funcionários para melhorar a segurança do paciente; } \\
\text { reconhece a participação dos funcionários para procedimentos de } \\
\text { melhoria da segurança do paciente. }\end{array}$ & $76 \%$ & Forte \\
\hline D3 & $\begin{array}{l}\text { Aprendizado organizacional - melhoria contínua: avalia a existência do } \\
\text { aprendizado a partir dos erros que levam a mudanças positivas e avalia a } \\
\text { efetividade das mudanças ocorridas }\end{array}$ & $76 \%$ & Forte \\
\hline D4 & $\begin{array}{l}\text { Adequação de profissionais: avalia se os funcionários são capazes de } \\
\text { lidar com sua carga de trabalho e se as horas de trabalho são adequadas } \\
\text { para oferecer o melhor atendimento aos pacientes. }\end{array}$ & $45 \%$ & Frágil \\
\hline D5 & $\begin{array}{l}\text { Respostas não punitivas aos erros: avalia como os funcionários se } \\
\text { sentem com relação aos seus erros, se eles pensam que os erros } \\
\text { cometidos por eles possam ser usados contra eles e mantidos em suas } \\
\text { fichas funcionais. }\end{array}$ & $40 \%$ & Frágil \\
\hline D6 & $\begin{array}{l}\text { Retorno da informação e comunicação sobre o erro: avalia a percepção } \\
\text { dos funcionários no hospital se eles relatam } \\
\text { os erros que ocorrem, se implementam mudanças e discutem } \\
\text { estratégias para evitar erros no futuro. }\end{array}$ & $71 \%$ & Moderado \\
\hline D7 & $\begin{array}{l}\text { Abertura à comunicação: Avalia se os funcionários do hospital } \\
\text { conversam livremente sobre os erros que podem afetar o paciente e se } \\
\text { eles se sentem livres para questionar os funcionários com maior } \\
\text { autoridade. }\end{array}$ & $60 \%$ & Moderado \\
\hline \multicolumn{4}{|c|}{ Nível da organização hospitalar } \\
\hline D8 & $\begin{array}{l}\text { Apoio da gerência para a segurança do paciente: avalia se a } \\
\text { administração e gestão do hospital propiciam um clima } \\
\text { de trabalho que promove a segurança do paciente demonstra que } \\
\text { a segurança do paciente é prioritária. }\end{array}$ & $76 \%$ & Forte \\
\hline
\end{tabular}


D9 Passagens de plantão/turno e transferências internas: avalia se informações importantes sobre o cuidado aos pacientes é transferida através das unidades do hospital e durante as $45 \%$ Frágil mudanças de plantão ou de turno.

D10 Trabalho em equipe entre as unidades: avalia se as unidades do hospital cooperam e coordenam-se entre si para prover um cuidado de alta qualidade para os pacientes.

$56 \%$

Moderado

Medidas de resultado de itens múltiplos

D11 Frequência de eventos notificados: relato de possíveis problemas de segurança do paciente e de eventos identificados ou erros percebidos e corrigidos antes que esses afetassem o paciente.

D12 Percepção geral da segurança do paciente: avalia os sistemas e procedimentos existentes na organização de saúde para evitar a ocorrência de erros e a ausência de problemas de segurança do paciente nos hospitais

\begin{tabular}{lll}
\hline Medidas de resultado ítens únicos & & \\
\hline $\begin{array}{l}\text { Grau geral de avaliação de segurança do paciente } \\
\text { Notificação de eventos adversos e/ou não conformidades em } 12 \\
\text { meses }\end{array}$ & $87 \% *$ & Forte \\
\hline
\end{tabular}

\footnotetext{
* Somados os percentuais de respostas 'Muito Boa' e 'Excelente'

** medida em faixas: entre zero até 5 notificações em 12 meses

Fonte: Dados primários.
}

Observa-se que as dimensões que apresentaram percentuais médios mais positivos para a cultura de segurança foram 'Aprendizado Organizacional - Melhoria Contínua', 'Apoio da Gerência para a Segurança do Paciente' e 'Frequência de Relato de Erros' e 'Expectativa sobre o Supervisor/Chefe e Ações Promotoras da Segurança do Paciente', todas com percentual de $76 \%$.

Nas medidas de resultados de itens únicos e de itens múltiplos, observam-se percentuais de $61 \%$ na dimensão 'Percepção Geral de Segurança do Paciente' e 87\% quanto ao 'Grau geral de Avaliação de Segurança do Paciente', o que evidencia a predominância de respostas positivas quanto ao reconhecimento de preocupações e ações voltadas para a segurança dos pacientes. A dimensão 'Notificação de Eventos de Segurança nos últimos 12 meses' obteve 89\% de respostas positivas para 'nenhuma notificação'. Todavia isso não garante a inexistência de eventos adversos, pois o instrumento não mensura subnotificações.

As percepções positivas de respostas (acima de 76\%) nas dimensões 'Aprendizado Organizacional', 'Apoio da Gestão Hospitalar para Segurança do Paciente', 'Frequência de Eventos Relatados' e 'Expectativas e Ações de Promoção de Segurança dos Supervisores / Gerentes’ evidenciaram-se como dimensões fortes para a cultura de segurança do paciente no hospital pesquisado. Disso depreende-se que a maioria dos respondentes (76\%) reconhecem a ocorrência de aprendizagens de comportamentos para garantir maior segurança aos pacientes e também percebem ações voltadas a segurança do paciente por parte das lideranças (supervisores/chefes) (76\%).

Além disto, no que se refere a gestores, os respondentes percebem preocupação destes com a segurança do paciente, expressa tanto nas expectativas e ações quanto no comportamento de apoio da gerência. Por esses percentuais evidenciam-se ainda percepções de reconhecimento de espaços organizacionais para a aprendizagem contínua de comportamentos que favoreçam a segurança do paciente. Isso parece reforçado pelos respondentes quanto a frequência de relatos de erros. Ademais, a explicitação dos riscos e problemas se torna uma habilidade crítica para o fortalecimento da cultura de segurança do paciente, individual e organizacionalmente, por meio da aprendizagem organizacional (Façanha, 2019).

De acordo com Bethel (2020), aprendizado organizacional reflete a capacidade dos integrantes de um grupo ou organização em examinar e revisar suas formas de trabalhar para aprimorar tarefas e processos, o que facilita a ampliação do 
grau de satisfação das pessoas com o seu trabalho e com a organização. Parece certo que encobrir erros ou buscar culpados, tende a ampliar a magnitude do problema. Ou seja, a cultura de segurança se fortalece e intensifica quando falhas são analisadas e suas origens identificadas.

A dimensão ‘Apoio da Gestão Hospitalar para Segurança do Paciente’ avalia se a administração e gestão do hospital propiciam um clima de trabalho que promove a segurança do paciente e demonstra que a segurança do paciente é prioritária. Encontrou-se $76 \%$ dos participantes do HG que responderam positivamente ao apoio da gestão. A gestão das pessoas parece exercer um papel relevante para a promoção de um ambiente que propicie aos funcionários criarem vínculos afetivos positivos com o trabalho e com a organização (Kanan \& Marcon, 2017) e estes vínculos tendem a contribuir para o clima organizacional e também para o bom desempenho no trabalho. No que se refere a segurança do paciente, entende-se que está relacionado também ao bom desempenho dos trabalhadores da saúde, juntamente com o bom desempenho da gestão (Oliveira et al., 2020).

$\mathrm{Na}$ dimensão 'Frequência de Relato de Eventos Adversos ou Não-conformidades' observa-se que os respondentes evidenciaram níveis de concordâncias importantes, pois situam-se próximos a 76\%. Isso parece indicar maior convicção dos respondentes quanto a ocorrência de relato de eventos que podem influenciar a segurança do paciente no hospital. Em outros termos, as percepções dos respondentes denotam a preocupação de notificar eventos adversos e/ou erros de processos (passíveis de) ou antes de gerarem danos aos pacientes. Sabe-se que o monitoramento de eventos adversos e/ou nãoconformidades (como exemplos: os quase-acidentes ou incidentes) é reconhecido como uma contribuição positiva para a aprendizagem coletiva para a segurança (Madalozzo \& Zanelli, 2016).

A respeito das 'Expectativas e Ações de Promoção de Segurança dos Supervisores/Gerentes', onde é avaliado se estes consideram as sugestões dos funcionários para melhorar a segurança dos pacientes e se reconhece a participação dos funcionários para procedimentos de melhoria da segurança do paciente, obteve-se $76 \%$ de respostas positivas. Tal achado vai ao encontro do que Pascuci, Meyer Junior e Crubelatte (2017) afirmam: para ocorrer o funcionamento adequado de uma organização hospitalar, é necessário que todas as partes (ou níveis) que a compõem estejam integradas de forma a conciliar ações e resultados através do esforço cooperado de seus membros. Assim como a gestão desenvolve expectativas quanto aos seus trabalhadores, estes, por sua vez, também possuem expectativas relacionadas a quem os coordena. Im e Park (2018) destacam que potencializar as lideranças é uma parte importante do desenvolvimento de cultura de segurança do paciente nos hospitais.

Ressalta-se que os autores do instrumento HSOPSC definem que percentuais moderados são aqueles que se situam entre $51 \%$ e $74 \%$. Neste viés, as dimensões 'Trabalho em Equipe dentro das Unidades' (71\%), 'Retorno da Informação e comunicação sobre o erro' (71\%) 'Abertura da Comunicação' (60\%) e 'Trabalho em Equipe entre as Unidades Hospitalares' (56\%) "evidenciam percentuais moderados da cultura de segurança. Embora com percentuais elevados de respostas positivas, como é o caso das duas primeiras dimensões com mais de 70\%, segundo os critérios de classificação, elas não podem ser consideradas como dimensões fortes para a cultura de segurança.

Todavia, a maioria dos respondentes reconhece a ocorrência de trabalho em equipe dentro das unidades (71\%). Ainda que com potencial moderado, tal fato reforça a capacidade de cooperação para trabalhar em equipe como aspecto importante à concretude do cuidado seguro e de qualidade para os pacientes (Reis, 2013). Em vista deste argumento, depreende-se que a percepção de apoio social reconhecida entre pares tende a melhor a qualidade das relações interpessoais nas equipes de trabalho e contribui para que os melhores resultados sejam entregues.

Na dimensão 'Retorno da Informação e Comunicação sobre o Erro' (71\%), o conjunto das informações manifestas pelos participantes possibilita traduzir suas percepções de eficácia no processo comunicacional, quando associado às possibilidades de erro, algo que converge à minimização de danos ao paciente e ao sistema (Marques, et al., 2019). Portanto, em conformidade com os resultados apurados, nas percepções dos respondentes, a comunicação que se estabelece entre as 
lideranças e os profissionais parece determinar em boa medida comportamentos e reações (des)favoráveis em relação à comunicação de erros que prevalecem no ambiente laboral (Ederer, König-Bachmann, Romano, Knobloch, \& Zenzmaier, 2019).

A comunicação nas organizações hospitalares e o trabalho das equipes interdisciplinares de saúde representam determinantes à qualidade e à segurança na assistência aos pacientes e seus familiares, conforme a Joint Commission International (JCI, 2013). Todavia, os profissionais necessitam vivenciar relações de confiança com seus pares e gestores para que a comunicação flua positivamente em todas as direções. Esquemas de flexibilização contribuem para a consolidação de canais de comunicação efetivos entre diferentes níveis hierárquicos, algo que converge para que os erros sejam relatados, sem, contudo, macular as relações estabelecidas (Lindberg Wahlén \& Pellgaard, 2020). Esses entendimentos parecem se refletir no conjunto de elementos da dimensão 'Abertura à Comunicação' com percentual de respostas positivas de $60 \%$, mas não suficientes para ser considerada como dimensão forte da cultura de segurança no local.

Por fim, na análise geral da dimensão 'Trabalho em Equipe entre as Unidades' é possível visualizar os percentuais de respostas positivas situado num nível moderado da escala (56\%). Em outros termos, depreende-se que os participantes reconhecem o potencial para o trabalho conjunto embora nem sempre possam constatá-lo na prática laboral. A partir disto, é possível questionar as possíveis razões que obstaculizam o efetivo trabalho em equipe entre as unidades.

Convém assinalar ainda, que nas medidas de resultados em itens únicos e múltiplos se observa $61 \%$ na dimensão 'Percepção Geral de Segurança do Paciente' e 61\% quanto ao 'Grau Geral de Avaliação de Segurança do Paciente' (todavia esse percentual alcance $87 \%$ quando somados os percentuais de respostas 'muito boa' e 'excelente), classificando-se como indicadores moderados de predição para a cultura de segurança do paciente. O que ocorre de modo similar a 'Notificação de Eventos de Segurança nos últimos 12 meses', com 55\% de respostas positivas para 'nenhuma notificação'.

Para os autores do instrumento HSOPSC, as dimensões com percentuais abaixo de 50\% são consideradas frágeis para a segurança do paciente. 'Dimensionamento de Profissionais' (45\%) avalia se os profissionais são capazes de lidar com a sua carga de trabalho e se as horas de trabalho são adequadas para oferecer o melhor atendimento ao paciente (Reis, 2013). Nessa interação entre produtividade e segurança, as pressões, em certa medida, podem ser consideradas como rotina, mas jamais irrelevantes. Esse entendimento é corroborado por Ugwu, Idike, Ibiam, Akwara e Okorie (2020), quando destacam que a pressão de trabalho está associada a uma menor conformidade com o comportamento de segurança no trabalho. Tal fato merece atenção quando o propósito é fortalecer a cultura de segurança dos pacientes.

A dimensão 'Mudança de Turno' com 45\% de respostas positivas pode indicar a necessidade de um monitoramento mais apurado nas trocas de plantão ou de turnos ao deixar evidente dúvidas quanto a influência da troca de turnos / plantões na segurança dos pacientes.

De modo similar, a dimensão 'Respostas Não Punitivas aos Erros', com 40\% de respostas positivas, demonstra ser frágil para a segurança do paciente. Considerado o contexto hospitalar como um ambiente crítico, a comunicação de um erro pode ser determinante para um desfecho satisfatório. Façanha (2019) a este respeito argumenta que ainda que notificar eventos adversos seja estratégia importante a ser utilizada à promoção de mudança de cultura de segurança do paciente, há espaço para melhorias neste sistema. A autora defende o entendimento que é preciso investir no sistema organizacional por meio melhorias constantes dos processos de trabalho e no comportamento dos profissionais diante de algum dano. Dessa forma, volta-se a importância da notificação de eventos adversos e a definição de políticas de recompensas e punições diante de erros como medidas para prevenção de sua ocorrência, para que seja possível o aprendizado com as falhas e a melhoria da gestão da cultura de segurança.

A seguir são apresentados os principais resultados nas dimensões que compõem cada grande área pesquisada no instrumento: o nível da unidade, o nível da organização hospitalar e as medidas de resultado de itens únicos e múltiplos. A 
ordem de apresentação segue a distribuição das dimensões nas respectivas áreas, conforme divisão dos itens do próprio instrumento de pesquisa.

\subsection{Nível da Unidade}

Na dimensão 'Expectativas e Ações de Promoção de Segurança dos Supervisores/Gerentes', 68\% dos participantes revelaram respostas positivas quanto ao recebimento de elogios por parte do supervisor/chefe quando vê um trabalho realizado de acordo com os procedimentos estabelecidos de segurança do paciente. Tal achado se reveste de significado quando se tem conhecimento de que reconhecer o trabalho realizado pelo funcionário pode afetar significativamente seu nível de desempenho, além de contribuir para atingimento das metas, procedimentos e diretrizes estabelecidos pela gestão (El Masri \& Suliman, 2019).

Ao avaliar se o supervisor/chefe considera as sugestões dos profissionais para a melhoria da segurança do paciente, $81 \%$ dos participantes responderam positivamente. A este respeito, encontra-se em Singh e Vanka (2019) que a voz do funcionário não pode ser ignorada. Conforme os autores, ouvir os funcionários enseja vantagens e, portanto, deve ser comportamento priorizado, promovido e praticado.

No que se refere ao aumento da velocidade das ações sempre que a pressão aumenta, mesmo que isso signifique 'pular etapas', verifica-se que 73\% dos participantes discordam desta afirmação. Isso indica que a maioria dos respondentes percebe a importância de agir de acordo com os procedimentos seguros e necessários, próprios de suas atividades, independentemente de uma possível pressão temporal.

Outro resultado obtido foi que $82 \%$ dos participantes discordam da afirmativa de que o seu supervisor/chefe não dá atenção suficiente aos problemas de segurança do paciente que acontecem repetidamente. Depreende-se assim que a preocupação da liderança com a segurança dos pacientes é reconhecida pelos funcionários, algo que contribui para a manutenção das estratégias de cuidado. É válido inferir que os comportamentos de liderança têm um impacto significativo na cultura de segurança do paciente (Ree \& Wiigs, 2020). Em outros termos, a educação e treinamento auxiliam no desenvolvimento de uma cultura de segurança do paciente e incluem também ações de liderança, relatórios, aprimoramento da cultura hospitalar e comunicação emocional (Im \& Park, 2018).

Na dimensão 'Aprendizado Organizacional - Melhoria Contínua', os resultados apontam mais respostas positivas. Pela percepção dos respondentes há esforços ativos para melhorar a segurança do paciente (90\%), aprendizagens a partir de erros a ponto de promover mudanças $(62 \%)$ e avaliações da efetividade dessas mudanças implementadas para melhorar a segurança do paciente (77\%). Esses resultados podem evidenciar aprendizado organizacional e os efeitos decorrentes de pessoas que sabem lidar com os seus erros. Afinal, a capacidade de aprender com os erros e promover melhorias de processos e tarefas por parte dos membros das equipes constitui fator decisivo para o fortalecimento da segurança dos pacientes (Reis, 2013; Simsekler et al., 2020). Reforçam esse entendimento Simsekler et al. (2020) quando evidenciam que para que ocorram aprendizagens a partir de eventos adversos faz-se necessário existir um clima de confiança que permita explicitações de erros, onde as pessoas têm evidências de que não serão punidas.

$\mathrm{O}$ 'Trabalho em Equipe dentro das Unidades' é percebido por comportamentos de apoio uns aos outros (68\%), de colaboração quando há muito trabalho a ser feito rapidamente (77\%) e pela ajuda dos profissionais às áreas sobrecarregadas da unidade $(61 \%)$. Destaca-se a afirmação de que as pessoas se tratam com respeito na unidade com o maior percentual de concordância em toda dimensão (78\%). Os resultados evidenciam também, os itens de "colaboração quando há muito trabalho a ser feito rapidamente para concluí-lo devidamente" e de "tratamento de respeito entre as pessoas na unidade" com percentuais de respostas positivas acima de 75\%. A este respeito é interessante observar os resultados do estudo de BuljacSamardžić e van Woerkom (2018) que ressaltam o suporte da equipe positivamente relacionado à percepção da qualidade do 
cuidado e negativamente relacionado a erros de medicação. Os autores reforçam ainda, que a carga de trabalho percebida tem efeito negativo na percepção da qualidade do cuidado.

O estudo realizado por Cheikh et al. (2016) com a utilização do instrumento HSOPSC à coleta de dados evidencia resultados semelhantes ao presente estudo: apoio e ajuda entre funcionários como comportamentos intra-setores, embora com percentuais menores quando os construtos são avaliados entre equipes de diferentes setores. Os autores citam estudos com resultados semelhantes realizados nos Estados Unidos (80\% para o trabalho intra-setores e 58\% para o trabalho em equipe entre os setores) e na Arábia Saudita com uma diferença significativamente grande de 57\% entre o trabalho em equipe no serviço e entre os serviços.

No que se refere a dimensão 'Abertura à Comunicação', é pertinente considerar que um importante obstáculo à comunicação e ao trabalho em equipe é o silêncio organizacional e a relutância e temor dos profissionais em expressar suas percepções, opiniões ou entendimentos a respeito do que efetivamente acontece em seu contexto de trabalho (Nogueira \& Rodrigues, 2015). Nestes termos, de modo oposto a esta consideração, é significativo aos gestores do hospital em estudo tomar conhecimento de que os profissionais sob sua responsabilidade percebem e declaram a liberdade que têm para se manifestar ou se posicionar ao identificar algo que pode afetar negativamente o cuidado ao paciente (77\%), bem como que predominam percepções daqueles que sentem-se à vontade para questionar as decisões ou ações dos seus superiores (48\%), seguidos de $31 \%$ no percentual de respostas neutras.

Tal resultado assume maior vulto, quando se considera que a expressão oral é um poderoso estímulo para que as pessoas organizem suas ideias e reflitam sobre as várias possibilidades de resolução de conflitos, escolhas ou decisões (Marques, Germana \& Kassandra, 2019), situações estas que podem influenciar os resultados de seu trabalho.

Uma vez que parece existir no hospital em estudo preocupação com a abertura da comunicação, cabe registar que não é de todo desconsiderada a existência de informações veladas por parte dos gestores, pois se todas as informações forem de livre acesso, as consequências poderão ser mais nocivas que benéficas (McCorry \& Mason, 2020).

Justificado pela importância de práticas preventivas, reitera-se que processos comunicativos ineficazes se associam diretamente à possibilidade da ocorrência de erros e, consequentemente, a possíveis danos irreversíveis ao paciente (Marques et al., 2019). Cabe, portanto, aos dirigentes manter ou mesmo, ampliar a confiança existente na comunicação entre os profissionais de todos os níveis hierárquicos, pois tal condição parece ser inversamente proporcional a ocorrência de erros e problemas associados à segurança dos pacientes.

A comunicação perpassa a vida das pessoas e integra seu cotidiano, independentemente de vontade, de concordância ou de autorização. Portanto, está presente em todas as possibilidades de interação social. Não seria diferente no contexto das organizações hospitalares. No hospital em estudo, ao avaliarem alguns aspectos importantes da comunicação entre pessoas, equipes e chefias, como por exemplo o 'Retorno da Informação e Comunicação sobre o Erro', os respondentes produziram resultados expressivos às questões que o indicador avalia.

Pode-se observar, por exemplo, que a percepção de abertura no processo comunicacional (61\%), as informações sobre os erros que acontecem nas unidades (72\%) e as discussões sobre meios de prevenir erros evitando que se repitam (79\%), são condições que espelham relações de troca, tornam comum a mensagem a todos e fortalecem a consonância e conformidade desta entre quem a emite e quem a recebe. Uma vez que estão estabelecidas tais condições, a ocorrência de erros parece contingenciada, ao menos nas percepções dos respondentes. Isto é reconhecido por Alenezi et al. (2019), quando asseguram que os líderes hospitalares devem criar um ambiente de trabalho onde erros possam ser relatados sem restrição e medo de repreensão.

A título de prevenção, registra-se ainda, que a ocorrência de status moderado no indicador 'Retorno da Informação e Comunicação sobre Erros' pode haver comprometimento da segurança do paciente, uma vez que a minimização ou ocultação 
de eventos ou erros a serem relatados pode se constituir em prática recorrente (Santiago \& Turrini, 2015). De fato, erros detectados e imediatamente relatados são cruciais às intervenções corretivas ou mitigadoras e à adoção de medidas preventivas ao restabelecimento das condições vitais do paciente e à redução dos efeitos nocivos do erro para ele (Tomazoni, Rocha, Kusahara, Souza \& Macedo, 2015), algo que se depreende ocorrer no hospital geral. Nessa direção, o percentual expressivo de respostas positivas à questão "nesta unidade, discutimos meios de prevenir erros evitando que eles aconteçam novamente" (79\%) reforça os resultados do indicador que refere a ocorrência de aprendizagem organizacional a partir dos erros.

Dado o exposto, registra-se que os resultados apurados são consistentes com as necessárias ações fortalecedoras da cultura de segurança e se revestem de tamanha importância que a JCI considera a comunicação eficaz como o Segundo Objetivo Internacional de Segurança do Paciente (JCI, 2013).

Baixas adesões a comunicação podem ainda estar relacionadas à 'Abordagens Punitivas aos Erros'. Os resultados evidenciam o predomínio de percepções discordantes para o item "quando um evento é notificado, parece que o foco recai sobre a pessoa e não sobre o problema" (46\%). Todavia, para os itens "os profissionais consideram que seus erros podem ser usados contra eles" (31\%) e "os profissionais se preocupam que seus erros sejam registrados em suas fichas funcionais" (15\%), os percentuais de discordância diminuem, o que pode revelar certo grau de insegurança dos respondentes quanto às formas como seriam tratados se cometessem erros. Observa-se aqui a necessidade de rever os procedimentos relativos à ocorrência de erros, como são relatados, analisados e classificados para serem sanados. Para fortalecer a cultura de segurança do paciente é necessário criar condições no cotidiano de trabalho que permitam e estimulem os profissionais de saúde identificar e explicitar erros cometidos e suas causas, com vistas a criação de estratégias de prevenção a segurança do paciente (Santiago \& Turrini, 2015; Tomazoni et al., 2015).

Práticas de gestão que considerem a adoção de 'Respostas não Punitivas ao Erro' são essenciais em todas as organizações de trabalho, mas se revestem de maior significado na assistência à saúde. Isto porque não há garantias de que a identificação de 'culpados' promova a diminuição dos erros. Por oposto: derivam desta medida as subnotificações, condição que obstaculiza a implantação de protocolos que previnam erros (Abreu et el., 2019). A este respeito, se observa nas respostas dos profissionais do hospital em estudo que erros são percebidos como possibilidade de melhorar as estratégias preventivas, bem como que a adoção de medidas punitivas a eles é incomum naquele contexto.

Com relação a dimensão 'Adequação do Quantitativo de Profissionais', de modo individualizado, observa-se que na questão "temos pessoal suficiente para dar conta da carga de trabalho" parece não haver consenso entre os respondentes do hospital, pois, embora o percentual de concordância seja de $45 \%$, os discordantes chegam a $34 \%$ e os neutros em $21 \%$. Isso pode sugerir a necessidade de analisar a carga de trabalho em relação ao efetivo médio de funcionários quando a preocupação é ampliar o cuidado seguro e com qualidade para os pacientes.

$\mathrm{Na}$ questão "os profissionais desta unidade trabalham mais horas do que seria o melhor para o cuidado do paciente", 45\% dos respondentes do hospital discordam ou discordam totalmente de tal afirmação, sugerindo não haver necessidade de trabalhar mais horas do que trabalham para assegurar cuidado com os pacientes. Todavia os percentuais de "não concordo, nem discordo" chegam a 32\%, o que revela a necessidade de compreender de modo mais aprofundado as dúvidas de vários respondentes quanto a essa afirmação.

A este respeito é pertinente considerar que as demandas da equipe hospitalar vêm crescendo nos últimos anos. Afirmam Sturm et al. (2019) que a carga de trabalho é fator decisivo para o cuidado com o paciente. Os autores asseguram ainda, que horas extras, bem como demais aspectos definidores de 'carga de trabalho' correlacionam-se claramente com o estresse e a tensão relacionados ao trabalho. Este é o mesmo entendimento de Al Ma'mari, Sharour e Omari (2020), pois em seu estudo encontraram relação significativa entre ambiente de trabalho, exaustão emocional, despersonalização, realização 
pessoal e cultura organizacional de segurança do paciente, sendo que a carga de trabalho representada pela fadiga, ambiente de trabalho, exaustão emocional, despersonalização e realização pessoal influenciaram a segurança geral do paciente.

$\mathrm{Na}$ questão "utilizamos mais profissionais temporários terceirizados do que seria desejável para o cuidado do paciente", $60 \%$ dos respondentes discordam ou discordam totalmente dessa afirmação, o que indica não haver necessidade de contar com profissionais além do efetivo da unidade para assegurar cuidados com os pacientes nas percepções dos respondentes.

$\mathrm{Na}$ afirmação "nós trabalhamos em situações de crise, tentando fazer muito e muito rápido", os respondentes do hospital apresentaram posições proporcionais entre concordâncias (37\%), dúvida (32\%) e discordâncias (31\%). Isso evidenciou percepções divergentes e, de certa maneira, até antagônicas dos respondentes quanto às formas de trabalho em situações de crise. Embora o cotidiano de um hospital deva ser conduzido por princípios de resolutividade em função da dinamicidade das tarefas, fazer muito e muito rápido pode sacrificar normas de cuidado e segurança, não apenas dos pacientes como também dos funcionários.

Concordar em querer responder a muitas tarefas ao mesmo tempo e muito rápido pode comprometer o cuidado com o paciente. Os respondentes indicaram valores de concordância inferior nessa afirmação. Isso parece revelar percepções concordantes de que, no hospital, não há evidências de que, em situações de crise, os profissionais queiram fazer tudo e muito rápido. Nesse sentido depreende-se que tal fato representa um aspecto favorável para não comprometer a segurança do paciente.

\subsection{Nível da Organização Hospitalar}

Esta área é composta por três dimensões: 'Apoio da Gestão Hospitalar à Segurança do Paciente', 'Trabalho em Equipe entre as Unidades' e 'Passagem de Plantão ou de Turno/Transferências Internas'. No primeiro, se a direção do hospital propicia um clima de trabalho que promove a segurança do paciente, $85 \%$ dos participantes responderam positivamente. $\mathrm{Na}$ percepção dos respondentes, portanto, existe um ambiente favorável no que se refere a promoção de segurança por parte da direção. O ítem "as ações da direção do hospital demonstram que a segurança do paciente é uma prioridade principal" obteve também um elevado percentual, com $82 \%$ de respostas positivas. A partir das respostas considera-se que a direção do hospital em estudo tem sido um fator positivo para que as ações relacionadas à segurança sejam respeitadas e efetivadas.

Os resultados parecem indicar que para a direção é estratégico "cuidar" das pessoas, no caso aqui, dos pacientes e também do bem-estar dos trabalhadores. A gestão é entendida como um conjunto de técnicas destinadas a racionalizar e otimizar o funcionamento das organizações, e é considerada como um sistema de organização do poder. Para dar conta de tantas exigências relacionadas com pessoas, compete à direção e também a gestão de pessoas, planejar, organizar e implementar políticas que as valorizem (Kanan \& Marcon, 2017).

O último item desta dimensão questiona se a direção do hospital só parece interessada na segurança do paciente quando ocorre algum evento adverso. Foi identificado que $61 \%$ dos participantes discordam dessa afirmação, o que indica predomínio de percepções que reconhecem interesse da direção não só na ocorrência de adversidades.

Numa primeira interpretação, observa-se o predomínio de percepções favoráveis a existência de trabalho em equipe entre as unidades. Todavia, ao examinar as questões de modo individualizado percebe-se que, embora os percentuais de discordância (46\%) sejam praticamente o dobro sobre os de concordâncias (24\%) o que indica percepções favoráveis dos respondentes à cultura de segurança, a questão "as unidades do hospital não estão bem coordenadas entre si" evidencia percentuais de indecisão que merecem ser considerados (30\%). Isso parece expressar dúvidas dos respondentes quanto a existência de coordenação entre as diferentes unidades do hospital que, no cotidiano de cuidados com o paciente pode sugerir gaps quanto ao alinhamento de processos de trabalho entre as unidades que podem comprometer sua segurança. 
Em contrapartida, embora 30\% seja o percentual de indecisão, 58\% dos respondentes concordam ou concordam totalmente com a afirmação de que existe boa cooperação entre as unidades do hospital. Isso pode expressar o reconhecimento de cooperação entre as unidades, todavia de modo não suficientemente coordenado entre elas. Tais percentuais indicam necessidade de revisão nesse sistema.

$\mathrm{Na}$ questão "muitas vezes é desagradável trabalhar com profissionais de outras unidades do hospital" observa-se o predomínio de discordâncias dos respondentes quanto a essa afirmação (50\%), o que pode denotar menos conflitos nas relações entre as unidades. Todavia o percentual de neutralidade (não concordo, nem discordo) merece consideração por apontar incerteza de resposta, o que se constitui num dado a ser examinado.

Por outro lado, o percentual de concordâncias entre os respondentes é maior na afirmação de que "as unidades do hospital trabalham bem em conjunto para prestar o melhor cuidado aos pacientes" $(70 \%)$, o que pode indicar reconhecimento dos respondentes da capacidade dos membros das unidades do hospital para trabalhar em conjunto em favor do paciente. Resta sugerir reflexões sobre possíveis razões que possam estar obstaculizando o efetivo trabalho em equipe entre as unidades.

Nesse cenário é possível hipotetizar duas situações quanto à dimensão "trabalho em equipe entre as unidades":

a) a percepção de uma fragilizada coordenação entre as unidades do hospital por parte dos respondentes, e

b) a percepção de aumento da cooperação entre as áreas quando fica evidente a importância da ação conjunta para prestar melhor a segurança dos pacientes.

Os percentuais evidenciam baixos níveis de concordância na média geral da dimensão 'Passagem de Plantão ou de Turno / Transferências Internas'. Comparando-se todas as questões do indicador, embora a média de concordâncias aumente quanto ao reconhecimento de que as mudanças de plantão ou de turno sejam problemáticas para os pacientes (55\%), os resultados nas outras 3 questões evidenciam graus de concordâncias menores:

a) O processo de cuidado é comprometido quando um paciente é transferido de uma unidade para outra (41\%).

b) É comum a perda de informações importantes sobre o cuidado do paciente durante as mudanças de plantão ou de turno $(44 \%)$.

c) Com frequência ocorrem problemas na troca de informações entre as unidades do hospital (41\%).

Índices menores de concordância nesses itens significam que os respondentes não percebem evidências fortes de que, no hospital, o cuidado com a segurança do paciente fique comprometido quando é preciso transferência para outra unidade, ou que ocorram perdas de informações sobre os pacientes ou ainda que aconteçam problemas na troca de informações entre as unidades do hospital. Todavia, tais resultados parecem apontar para a necessidade de um monitoramento mais apurado nas trocas de plantão ou de turnos ao evidenciarem dúvidas quanto a influência da troca de turnos / plantões na segurança dos pacientes.

\subsection{Medidas de Resultados de Itens Únicos}

Esta dimensão evidencia o 'Grau Geral de Avaliação de Segurança do Paciente' e 'Número de Eventos Notificados nos últimos 12 meses'. O estudo revelou que 87\% dos participantes avaliaram o grau geral de avaliação segurança do paciente entre 'muito bom' e 'excelente', o que denota um clima favorável à segurança do paciente, reforçado pelo percentual de $61 \%$ quanto a percepção geral da segurança do paciente. Porém, Madalozzo e Zanelli (2016), em estudo que revisou a literatura sobre clima e cultura de segurança, referem que o clima pode ser um indicador de desempenho de segurança, mas é no nível de pressupostos básicos que se localiza o núcleo da cultura de segurança. Em outros termos, percepções e atitudes revelam mais sobre o clima do que sobre a cultura de segurança, que é expressa em comportamentos com base em valores e crenças inconscientemente enraizados. 
De acordo com a Tabela 2, embora os percentuais de itens 'Frequência de Eventos Notificados' apontem essa como uma dimensão forte para a segurança do paciente (76\%), a baixa notificação de eventos adversos e/ou não conformidades em 12 meses (89\% até 5 notificações) contradiz o percentual anterior. Disso depreende-se que as percepções dos respondentes parecem mais positivas sobre relatos de eventos adversos do que realmente o são no cotidiano (ao menos em doze meses), o que aponta a necessidade de incentivo às ações para a explicitação de eventos adversos, a fim de que possam ser conhecidos e mitigados.

\subsection{Medidas de Resultados de Itens Múltiplos}

Esta área avalia as dimensões 'Percepção Geral da Segurança do Paciente' e Frequência de Relatos de Evento. Os percentuais evidenciam uma percepção geral positiva, embora em níveis moderados, dos respondentes quanto à segurança do paciente (61\%) e se expressam nos seguintes resultados: Há 59\% de respondentes que discordam da afirmação de que 'erros graves só não ocorrem por acaso', 53\% reconhecem que 'a segurança do paciente jamais é comprometida por maior quantidade de trabalho a fazer', $62 \%$ discordam ou discordam totalmente que 'existem problemas de segurança do paciente em sua unidade', e 70\% concordam que 'seus procedimentos e sistemas são adequados para prevenir a ocorrência de erros'.

Como esta dimensão avalia o sistema e os procedimentos existentes na organização de saúde para evitar a ocorrência de erros e a ausência de problemas de segurança do paciente nos hospitais (Reis, 2013), os resultados evidenciam potencial de cuidado nas unidades e percepção ampliada para riscos, ao menos para os respondentes. Não há como avaliar, porém, se as respostas refletem critérios de defensividade por parte dos membros das equipes.

A dimensão "Percepção Geral da Segurança do Paciente" obteve $61 \%$ de respostas positivas. A segurança do paciente tende a estar relacionada com a prevenção de erros no atendimento médico-hospitalar, bem como a sua qualidade. Dado o exposto, entende-se necessário eliminar práticas de culpabilidade individual pelo erro ocorrido, para se investir em processos da assistência que permitam realizar as correções necessárias e criar mecanismos de controle e de aprendizagem que possibilitem antecipar os erros e assim preveni-los e a seus danos. Tal entendimento é reforçado por Andrade, Pereira e Nunes (2015), para quem a segurança do paciente pode ser um princípio e um qualificador do cuidado, e sua concretização ocorre a partir de medidas estruturais, organizacionais, gerenciais, de responsabilidade e de ações de formação profissional que contribuam para antecipar as possibilidades de erros.

De acordo com os percentuais apresentados na Tabela 2 referente a 'Frequência de Relato de Eventos', os respondentes:

a) concordam que eventos adversos e/ou erros de processos são notificados, mesmo sendo percebidos e corrigidos antes de afetar o paciente $(72 \%)$;

b) concordam que são notificados eventos adversos ou não-conformidades, mesmo não existindo riscos de danos aos pacientes ou ao processo $(74 \%)$;

c) concordam ainda que são notificados eventos adversos ou não-conformidades que poderiam causar danos aos pacientes ou aos processos (82\%), evidenciando o maior percentual de concordância no total neste indicador.

O elevado grau de concordância expresso nessa dimensão parece revelar percepções fortalecidas pelos respondentes quanto a ocorrência das notificações de eventos adversos e/ou não-conformidades, tanto em situações que poderiam causar danos ao paciente quanto naquelas em que o risco é mínimo ou inexistente. Disso depreende-se uma percepção de riscos ampliada no contexto de trabalho e o reconhecimento da importância de notificá-los, como restou evidente ainda nas avaliações que dizem respeito a dimensão 'Retorno de Informações e Comunicação sobre Erro'. Revela-se aqui aspectos que requerem a promoção de soluções que contribuam para fortalecer a segurança dos pacientes. 


\section{Considerações Finais}

O estudo aqui apresentado contribuiu para identificar com mais precisão as percepções dos funcionários sobre segurança dos pacientes em uma instituição hospitalar localizada no sul do Brasil, a partir da aplicação do instrumento HSOPSC, utilizado em diferentes países. Os principais resultados explicitaram 'Aprendizado Organizacional', 'Apoio da Gestão Hospitalar para Segurança do Paciente', 'Frequência de Eventos Relatados' e 'Expectativas Sobre o seu Supervisor/Chefe' e 'Ações Promotoras de Segurança do Paciente' como dimensões fortes para a segurança dos pacientes no hospital pesquisado. A fragilidade na predição dessa cultura ficou representada pelas dimensões 'Passagem de Plantão/Turno' e 'Respostas não Punitivas aos Erros'. Esses resultados destacam que as percepções de suporte da gestão e o reconhecimento de um ambiente propício para a explicitação, análise e aprendizagens com situações adversas, formam um prognóstico favorável em direção a sedimentação da cultura de segurança dos pacientes no hospital em tela. Conclui-se ainda que, reforçar estes aspectos promotores e intervir nos aspectos fragilizantes, bem como a análise detalhada de cada dimensão pesquisada, representem ações urgentes e necessárias a serem adotadas na organização para promover o efetivo cuidado integral em saúde.

A alta taxa de eventos adversos encontrada no estudo de Aranaz-Andrés (2011) em cinco países Latino-Americanos vem reforçando, há cerca de 10 anos, que a segurança do paciente representa um importante problema de saúde pública nos hospitais. Nestes termos, ainda que este seja um problema complexo, é urgente e necessário abordá-lo em seu grau de importância e repercussões para os profissionais do cuidado, para as instituições hospitalares, mas principalmente para os pacientes. Examinar a evitabilidade de eventos adversos é função precípua à adequação e excelência da atenção à saúde e assistência prestada. Por conseguinte, recomenda-se a gestores hospitalares que tenham como regra, aumentar a quantidade e a qualidade de suporte e orientação disponibilizados aos profissionais quando da identificação do evento adverso. Deste modo, sugere-se às instituições hospitalares e seus dirigentes, conforme o caso, a implementação, ampliação e/ou fortalecimento de políticas institucionais voltadas à promoção e adoção de ações que possam mitigar eventos adversos e ampliar aspectos enaltecedores e promotores da cultura de segurança do paciente em todos os níveis hierárquicos.

As mudanças na cultura de segurança do paciente se fundamentam em transformações procedimentais e estruturais que são construídas pela revisão de crenças e valores de todos os atores organizacionais quanto a perigos e eventos adversos para fortalecer neles o desejo efetivo de preservar a saúde integral (Simsekler et al., 2020; Oliveira et al, 2020). Tendo em vista os resultados obtidos nesse estudo, depreende-se como oportunas futuras pesquisas que aprofundem conhecimentos sobre as interfaces entre a cultura de segurança do paciente com a cultura de segurança no trabalho, uma vez que as relações de (des)cuidados ocorrem por meio de comportamentos humanos.

\section{Referências}

Abreu, I. M. et al. (2019). Patient safety culture at a surgical center: the nursing perception. Revista Gaúcha de Enfermagem, 40(spe), 1-8.

Agency for Healthcare Research And Quality (2004). Annual Report on Research and Management. http://www.ahrq.gov/about/annrpt04/

Alenezi, A., Pandaan, R. P. M., Almazan, J. U., Pandaan, I. N., Casison, F. S., \& Cruz, J. P. (2019). Clinical practitioners' perception of the dimensions of patient safety culture in a government hospital: A one-sample correlational survey. Journal of clinical nursing, 28(23-24), 4496-4503.

Al Ma'mari, Q. Sharour, L. A., \& Al Omari, O. (2020). Fatigue, burnout, work environment, workload and perceived patient safety culture among critical care nurses. British journal of nursing, 29(1), 28-34.

Andrade A. K. Y., Pereira S. V. E., \& Nunes D. C. (2015). A análise do conceito segurança do paciente: a visão evolucionária de Rodgers. Aquichan, 15(4), $521-528$.

Aranaz-Andrés, J. M. et al. (2011). Prevalência de eventos adversos em hospitais de cinco países latino-americanos: resultados do Estudo Iberoamericano de Eventos Adversos (IBEAS). BMJ Quality \& Safety, 20 (12), 1043-1051.

Bethel, K. L. (2020). An Evaluation of Organizational Culture: Its Influence on Security Culture: A Case Study. Doctor Dissertation, School of Business, Northcentral University. La Jolla, California, EUA. 
Buljac-Samardžić, M., \& van Woerkom, M. (2018). Improving quality and safety of care in nursing homes by team support for strengths use: A survey study. Plos one, 13(7), 1-16.

Cheikh, A. B., Bouafia, N., Mahjoub, M., Ezzi, O., Nouira, A., \& Njah, M. (2016). Patient's safety culture among Tunisian healthcare workers: results of a cross sectional study in university hospital. The Pan African medical journal, 24 (299), 1-8.

Costa, D.B., Ramos, D., Gabriel, C.S., \& Bernardes, A. (2018). Cultura de segurança do paciente: avaliação pelos profissionais de enfermagem. Texto \& Contexto - Enfermagem. 27(3), 1-9.

Doo, E. Y., \& Kim, M. (2020). Effects of hospital nurses' internalized dominant values, organizational silence, horizontal violence, and organizational communication on patient safety. Research in Nursing \& Health, 43(5), 499-510.

Ederer, C., König-Bachmann, M., Romano, I., Knobloch, R., \& Zenzmaier, C. (2019). Midwives' perception of patient safety culture: a qualitative study. Midwifery, 71, 33-4.

El Masri, N., \& Suliman, A. (2019). Talent Management, Employee Recognition and Performance in the Research Institutions. Studies in Business and Economics, 14(1), 127-140.

Façanha, T. R. S. (2019). Percepções de profissionais de saúde em uma instituição hospitalar: um enfoque bioético sobre cultura de segurança do paciente. Dissertação de Mestrado, Programa de Pós-Graduação em Bioética, Universidade de Brasília. Brasília, Brasil.

Im, S. A., \& Park, M. J. (2018). The Effects of Patient Safety Culture Perception and Organizational Commitment on Patient Safety Management Activities in General Hospital Nurses. Journal of Digital Convergence 16(6), 259-270.

Joint Commission Resources (2013). Joint Commission International Accreditation Standards for Hospitals. (5th ed.) Oak Brook, IL: Joint Commission Resources. www.jointcommissioninternational.org

Kanan, L. A. \& Marcon, S. R. A. (2017) Ambiente, Saúde e Gestão Humana de Recursos: Cartografia do Bem-Estar no Trabalho. In: Parreira, P.; Mónico, L.; Carvalho, C.. (Org.). Gestão de Pessoas nas Organizações. 75-96. Escola Superior de Enfermagem de Coimbra.

Lindberg Wahlén, J., \& Pellgaard, J. (2020). Factors in the nurse profession that have an impact on patient safety. Digital Scientific Archive.https://www.diva-portal.org/smash/record.jsf?pid=diva2\%3A1440749\&dswid=5100

Madalozzo, M. M. \& Zanelli, J. C. (2016). Segurança no Trabalho: a construção cultural dos acidentes e catástrofes no cotidiano das organizações. Curitiba: Juruá.

Marques, J. L., Germana, P.; Kassandra, I. (2019). Segurança do paciente: o envolvimento do paciente na segurança dentro da instituição. Revista Científica de Gestão Hospitalar, v. 1, n. 1, p. 129-134.

McCorry, L. K., \& Mason, J. (2020). Communication skills for the healthcare professional (2a ed.). Jones \& Bartlett Pub Incorporated.

Nogueira, J. W. S. \& Rodrigues, M. C. S. (2015). Comunicação efetiva no trabalho em equipe em saúde: desafio para a segurança do paciente. Cogitare Enfermagem, 20(3), 636-640.

Oliveira, J. L. C, Cervilheri, A. H., Haddad, M. C. L, Magalhães, A. M. M., Ribeiro, M. R. R., \& Matsuda L. M. (2020). Interface between accreditation and patient safety: nursing team perspectives. Revista Escola de Enfermagem USP, 54, 1-7.

Organização Nacional de Acreditação (2020). $6^{\circ}$ Encontro Nacional de Avaliadores. https://www.ona.org.br/mapa-de-acreditacoes

Paes, G. O., Braz, A. O., Sauthier, M., Oliveira, A. B. de, \& Stipp, M. A. C. (2020). Segurança do paciente e seu reconhecimento para a ética nos cuidados de Enfermagem. Research, Society and Development, 9(8), 1-14.

Pascuci, L. M., Meyer Júnior, V., \& Crubellate, J. M. (2017). Strategic Management in Hospitals: Tensions between the Managerial and Institutional Lens. BAR - Brazilian Administration Review, 14(2), 1-21.

Ree, E., \& Wiig, S. (2020). Linking transformational leadership, patient safety culture and work engagement in home care services. Nursing Open, 7(1), 256264.

Reis, C. T. (2013). A cultura de segurança do paciente: validação de um instrumento de mensuração para o contexto hospitalar brasileiro. Tese de doutorado, Programa de Pós-Graduação em Saúde Pública, Escola Nacional de Saúde Pública Sergio Arouca, Fundação Oswaldo Cruz. Rio de Janeiro, Brasil.

Reis C. T., Paiva S. G., Sousa P. (2018) The patient safety culture: a systematic review by characteristics of Hospital Survey on Patient Safety Culture dimensions. Int J Qual Health Care J Int Soc Qual Health Care, 30(9), 660-677.

Santiago, T. H. R. \& Turrini, R. N. T. (2015). Cultura e clima organizacional para segurança do paciente em Unidades de Terapia Intensiva. Revista da Escola de Enfermagem da USP, 49(spe), 123-130.

Singh, S., \& Vanka, S. (2019). Voice matters: why HR should listen to employee voice? Strategic HR Review. 18(6), 268-271.

Simsekler, M. C. E., Qazi, A., Alalamia, M. A., Ellahham, S., \& Ozonoff, A. (2020). Evaluation of patient safety culture using a random forest algorithm. Reliability Engineering and System Safety, 204, 1-9.

Sturm, H., Rieger, M. A., Martus, P., Ueding, E., Wagner, A., Holderried, M., Maschmann, J. \& WorkSafeMed Consortium. (2019). Do perceived working conditions and patient safety culture correlate with objective workload and patient outcomes: A cross-sectional explorative study from a German university hospital. PloS one, 14(1), 1-19. 
Research, Society and Development, v. 10, n.6, e55510616113, 2021

(CC BY 4.0) | ISSN 2525-3409 | DOI: http://dx.doi.org/10.33448/rsd-v10i6.16113

Tomazoni, A., Rocha, P. K., Kusahara, D. M., Souza, A. I. J. \& Macedo, T. R. (2015). Avaliação da cultura de segurança do paciente em terapia intensiva neonatal. Texto Contexto Enfermagem, 24(1), 161-169.

Ugwu, F. O., Idike, A. N., Ibiam, O. E., Akwara, F. A., \& Okorie, C. O. (2020). Transformational leadership and management safety practices: Their role in the relationship between work pressure and compliance with safety work behaviour in a health-care sector industry. Journal of psychology in Africa, 30(1), 18.

World Health Organization (2006). Quality of Care: a process for making strategic choices in systems Geneva: WHO https://www.who.int/management/quality/assurance/QualityCare_B.Def.pdf 